\title{
Relay Selection in Two-Way Full-Duplex Energy-Harvesting Relay Networks
}

\author{
Dexin Wang*, Rongqing Zhang*, Xiang Cheng ${ }^{\dagger}$, and Liuqing Yang* \\ * Department of Electrical and Computer Engineering, Colorado State University, Fort Collins, CO 80523. \\ $\dagger$ State Key Laboratory of Advanced Optical Communication Systems and Networks, \\ School of Electronics Engineering and Computer Sciences, Peking University, Beijing 100871, P. R. China \\ Emails: dxwang@engr.colostate.edu, rongqing.zhang@colostate.edu, xiangcheng@pku.edu.cn, lqyang@engr.colostate.edu
}

\begin{abstract}
In this paper, we investigate the optimization of the power splitting factor and the relay selection problem in two-way full-duplex (FD) relay networks, where the relays are wirelessly powered by harvesting a portion of the received signal power from the sources. To the best of the authors' knowledge, this is the first time that the two-way FD relays with simultaneous wireless and information transfer (SWIPT) capabilities are investigated. For each relay, we prove the quasi-convexity of the power splitting (PS) factor optimization and obtain the optimal PS factor in terms of the outage probability by linear search. We propose two relay selection schemes that minimize the outage probability and maximize the sum capacity respectively. The performance improvement over the random selection scheme and the allparticipate scheme is demonstrated by simulations.

Index Terms-Energy harvesting, full-duplex, relay selection, SWIPT, two-way relaying
\end{abstract}

\section{INTRODUCTION}

Green communications has been an attractive research area in recent years. By improving the energy efficiency of the entire system, it provides promising solutions for further development of communication systems such as wireless sensor networks (WSNs) and cellular networks. Among the technologies being studied, energy harvesting has the potential to significantly extend the lifetime of battery-powered devices and, in some cases, to eliminate the necessity of batteries. Besides traditional energy harvesting from sources such as solar, wind, vibration, and heat, a new solution is to exploit the energy carried by radio-frequency (RF) signals.

In the meantime, it is recognized that both energy and information are carried by the same wireless signals. Therefore, the technology of simultaneous wireless information and power transfer (SWIPT) was proposed [1]. In the literature, SWIPT schemes can be classified into two categories. In one category, the receivers are assumed to be able to extract information and harvest energy from the received signal simultaneously [1, 2]. However, practical circuits for energy harvesting from wireless signals are not yet able to directly process the information carried by the signals [3]. This triggers the study of the other category, in which the receivers adopt time switching (TS) or power splitting (PS) to coordinate the information processing (IP) and energy harvesting (EH). Either the total amount of time or the total power of the received signals is split between IP and EH.

SWIPT has been successfully applied to energy-constrained relays that can harvest energy from the received wireless signals. This type of relays operate mainly or exclusively based on the harvested energy [4]. TS-based relaying and PS-based relaying are two prevalent practical schemes. Each transmission cycle through the relay is divided into two phases in half-duplex (HD) EH relays. In the first phase, the relay receives signals from the source with SWIPT, in either TS or PS manner. In the second phase, the relay forwards the signals to the destination with the energy harvested in the first phase. With the advancement in self-interference cancellation techniques [5-7], full-duplex (FD) relay networks are gaining increasing interests due to their significant performance advantages over HD ones [8]. Several TS-based FD EH relay networks were proposed in [9-11]. These FD schemes utilize the spectral resource in a more efficient manner compared with HD ones. Nevertheless, the relay in all these FD schemes can only transmit and/or receive information during a fraction of each transmission cycle. Therefore, we proposed to further improve the relaying efficiency by fully exploiting the FD feature via PS-SWIPT in our previous work [12].

Relay selection (RS) is a practical solution to exploit the cooperative diversity in a wireless network where multiple relays are available. This topic has attracted considerable attention in academia [13-15]. The RS problem in energy harvesting relay networks is relatively new. Most existing researches were conducted on HD or one-way relay networks $[16,17]$. However, the unique characteristics of two-way FD EH relay networks bring new insights to the RS problem.

In this paper, we investigate the characteristics and performance of PS-based two-way full-duplex energy-harvesting (PS-TWFDEH) relay networks. Our contributions lie in the following three aspects: 1) To the best of the authors' knowledge, TWFDEH relay networks are investigated for the first time. We employ PS-based SWIPT at the EH relays, which can enable real FD information transmission in the investigated relaying network and improve the network spectrum efficiency effectively; 2) We prove that minimizing the outage probability is a quasi-convex optimization problem and that the optimal PS factor for each EH relay can be efficiently obtained by onedimensional search algorithms; and 3) Based on the optimized PS factor at each relay, we propose two RS schemes for the investigated TWFDEH relay network. Both RS schemes can achieve better performance than the AP scheme and the 
random RS scheme at high SNR.

\section{SySTEM MODEL}

As shown in Fig. 1, in this paper, we investigate a twoway full-duplex energy-harvesting (TWFDEH) amplify-andforward (AF) relay network. All the involved nodes, including two sources and $N$ relays, operate in FD mode, which means they transmit and receive signals simultaneously over the same frequency band. We assume that no direct link between the sources exists and the sources have perfect knowledge of all the channels, while each relay only knows the channels related to itself. The relay selection is processed at the sources and the decisions are then broadcast to the relays.

The relays receive signals from both sources. The selected relay amplifies the received signals and transmit the combined signals back to the sources simultaneously, so that the sources can extract the information sent from the other side. At the selected relay, the received signals are split according to a power ratio of $\rho_{j}:\left(1-\rho_{j}\right)$ for IP and $\mathrm{EH}$, respectively. The harvested energy is transferred to the batteries for temporary storage and then used to power the transmission circuits.

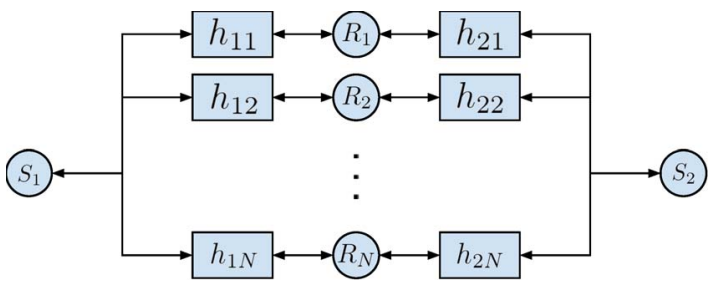

(a) System Diagram

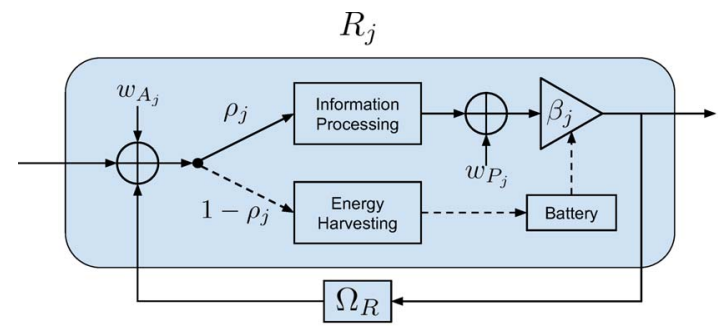

(b) Relay Node Diagram

Fig. 1: A full-duplex energy-harvesting relay network.

The channel response between $S_{i}(i=1,2)$ and $R_{j}(j=$ $1,2, \cdots, N)$ is denoted by $h_{i j}$. The self-interference due to the FD operation mode can be effectively suppressed by analog and digital self-interference cancellation methods [7]. However, the residual self-interference still plays a role in the performance of the network. In this paper, the residual selfinterference channel response of $S_{i}$ and $R_{j}$ are denoted as $\Omega_{S_{i}}$ and $\Omega_{R_{j}}$, respectively. Note that these are the equivalent channel response after the self-interference cancellation. Then, the received signal at $R_{j}$ is

$$
y_{R_{j}}[n]=\sum_{i=1}^{2} h_{i j} x_{S_{i}}[n]+\Omega_{R_{j}} x_{R_{j}}[n]+w_{A_{j}}[n]
$$

where $x_{R_{j}}$ denotes the transmit signal of $R_{j}$. The antenna noise and processing noise at the relays are represented by $w_{A_{j}}$ and $w_{P_{j}}$, whose distribution follows $\mathcal{C N}\left(0, \sigma_{A}^{2}\right)$ and $\mathcal{C} \mathcal{N}\left(0, \sigma_{P}^{2}\right)$, respectively.

If $R_{j}$ is selected to cooperate, the received signal $y_{R_{j}}$ is then split for IP and EH with a power ratio of $\rho_{j}:\left(1-\rho_{j}\right)$, $\rho \in[0,1]$, as described in

$$
\begin{aligned}
y_{\mathrm{IP}_{j}}[n] & =\sqrt{\rho_{j}} y_{R_{j}}[n] \\
y_{\mathrm{EH}_{j}}[n] & =\sqrt{1-\rho_{j}} y_{R_{j}}[n] .
\end{aligned}
$$

And $y_{\mathrm{IP}_{j}}$ is amplified and forwarded to the sources. Let $\beta_{j}$ denote the amplification factor at $R_{j}$. The transmit signal at $R_{j}$ is

$$
x_{R_{j}}[n]=\beta_{j}\left(y_{\mathrm{IP}_{j}}[n]+w_{P_{j}}\right) .
$$

Then the transmit power at $R_{j}$ is

$$
P_{R_{j}}=\mathbb{E}\left[\left|x_{R_{j}}\right|^{2}\right]=\beta_{j}^{2}\left(\rho_{j} \mathbb{E}\left[\left|y_{R_{j}}\right|^{2}\right]+\sigma_{P}^{2}\right) .
$$

The investigated EH relays do not rely on additional power sources. All the energy used for transmission at $R_{j}$ is harvested from $y_{\mathrm{EH}_{j}}$. This means that the transmit power equals to the harvested power on average. Therefore, we have

$$
P_{R_{j}}=\xi \mathbb{E}\left[\left|y_{\mathrm{EH}_{j}}\right|^{2}\right]
$$

where $y_{\mathrm{EH}_{j}}$ represents the signal for $\mathrm{EH}$ at $R_{j}$, and $\xi$ is the EH efficiency.

Therefore, we have

$$
\begin{aligned}
P_{R_{j}} & =\left(1-\rho_{j}\right) \xi \mathbb{E}\left[\left|y_{R_{j}}\right|^{2}\right] \\
& =\left(1-\rho_{j}\right) \xi\left(P_{S} \sum_{i=1}^{2}\left|h_{i j}\right|^{2}+\left|\Omega_{R_{j}}\right|^{2} P_{R_{j}}+\sigma_{A}^{2}\right) \\
P_{R_{j}} & =\frac{\left(1-\rho_{j}\right) \xi\left(P_{S} \sum_{i=1}^{2}\left|h_{i j}\right|^{2}+\sigma_{A}^{2}\right)}{1-\left(1-\rho_{j}\right) \xi\left|\Omega_{R_{j}}\right|^{2}}
\end{aligned}
$$

Combining (5) and (6), we obtain the value of $\beta_{j}$ as

$$
\beta_{j}=\sqrt{\frac{\xi\left(1-\rho_{j}\right) \mathbb{E}\left[\left|y_{R_{j}}\right|^{2}\right]}{\rho_{j} \mathbb{E}\left[\left|y_{R_{j}}\right|^{2}\right]+\sigma_{P}^{2}}} \approx \sqrt{\frac{\left(1-\rho_{j}\right) \xi}{\rho_{j}}} .
$$

The approximation is justified as $\rho_{j} \mathbb{E}\left[\left|y_{R_{j}}\right|^{2}\right] \gg \sigma_{P}^{2}$ in practical networks. Otherwise, the end-to-end SNR would be very small if the signal and interference could not dominate the received power at the relays.

The received signal at $S_{i}$ via the $j$ th relay is

$$
\begin{aligned}
y_{S_{i}}^{(j)}[n]= & h_{i j} x_{R_{j}}[n]+\Omega_{S_{i}} x_{S_{i}}[n]+w_{i j}[n] \\
= & h_{i j} \beta_{j}\left(\sqrt{\rho} y_{R_{j}}[n-1]+w_{P_{j}}[n-1]\right) \\
& +\Omega_{S_{i}} x_{S_{i}}[n]+w_{i j}[n] \\
= & h_{i j} \beta_{j} \sqrt{\rho}\left(h_{i j} x_{S_{i}}[n-1]+h_{\overline{i j}} x_{S_{\bar{i}}}[n-1]\right. \\
& \left.+\Omega_{R_{j}} x_{R_{j}}[n-1]+w_{A_{j}}[n-1]\right) \\
& +h_{i j} \beta_{j} w_{P_{j}}[n-1]+\Omega_{S_{i}} x_{S_{i}}[n]+w_{i j}[n]
\end{aligned}
$$

where $w_{i j}$ is the noise at $S_{i}$, which follows the distribution $\mathcal{C N}\left(0, \sigma_{S}^{2}\right) . \bar{i}$ denotes the index of the other source. The first term in (13) when expanded, $h_{i j}^{2} \beta_{j} \sqrt{\rho} x_{S_{i}}[n-1]$, can be canceled since all the components are known at $S_{i}$. 
Then the power of $y_{S_{i}}^{(j)}$ can be obtained as

$$
\begin{aligned}
\mathbb{E}\left[\left|y_{S_{i}}^{(j)}\right|^{2}\right]= & \beta_{j}^{2} \rho_{j}\left|h_{i j}\right|^{2}\left|h_{i j}\right|^{2} P_{i} \\
& +\left|h_{i j}\right|^{2}\left|\Omega_{R_{j}}\right|^{2} \beta_{j}^{2} \rho_{j} P_{R_{j}}+\left|h_{i j}\right|^{2} \beta_{j}^{2} \rho_{j} \sigma_{A}^{2} \\
& +\left|\Omega_{S_{i}}\right|^{2} P_{S}+\left|h_{i j}\right|^{2} \beta_{j}^{2} \sigma_{P}^{2}+\sigma_{S}^{2}
\end{aligned}
$$

where the expectation is taken with respect to source symbol realizations.

Only the first term on the right-hand side of (14) corresponds to the useful signal from the other source. Hence, the one-way end-to-end SINR is given in (15) and (16).

Therefore, the capacity of the link from $S_{\bar{i}}$ to $S_{i}$ is

$$
C_{S_{i}}=W \log _{2}\left(1+\gamma_{S_{i}}^{\left(j^{*}\right)}\right)
$$

where $j^{*}$ is the index of the selected relay.

\section{Power Splitting FACtor Optimization}

In this paper, we consider two important performance metrics for the relay selection problem, that is the outage probability and the ergodic sum capacity. The outage probability is defined as

$$
P_{\text {out }}=\operatorname{Pr}\left\{\min \left(C_{S_{1}}, C_{S_{2}}\right)<C_{\text {th }}\right\}
$$

where $C_{\text {th }}$ is the outage threshold below which the capacity is considered as unacceptable.

The ergodic sum capacity is defined as

$$
C_{E}=\mathbb{E}\left[C_{S_{1}}+C_{S_{2}}\right]
$$

in which the expectation is taken with respect to channel realizations.

\section{A. Minimum Outage Probability}

For each relay $R_{j}$, we can find the optimal PS factor that minimizes the outage probability if $R_{j}$ is selected. Note that for each channel realization, minimizing the outage probability is equivalent to maximizing $\min \left(C_{S_{1}}, C_{S_{2}}\right)$, which in turn is equivalent to maximizing $\min \left(\gamma_{S_{1}}^{(j)}, \gamma_{S_{2}}^{(j)}\right)$. Then, we have

$$
\rho_{j}^{*}=\underset{\rho_{j} \in[0,1]}{\arg \max } \min \left(\gamma_{S_{1}}^{(j)}, \gamma_{S_{2}}^{(j)}\right)
$$

Theorem 1. The objective function in (20), $\min \left(\gamma_{S_{1}}^{(j)}, \gamma_{S_{2}}^{(j)}\right)$, is quasi-convex with respect to the power splitting factor $\rho_{j}$ over the open interval $(0,1)$.

Proof: See the Appendix.

Apparently, neither $\rho_{j}=0$ nor $\rho_{j}=1$ is the minimizer of $J_{P_{\text {out }}}^{(j)}$. Therefore, Theorem 1 guarantees that we can find a $\rho_{j}$ that achieves the globally minimum outage probability for $\rho_{j} \in[0,1]$ by local search methods, such as the method of gradient descent or the Newton-Raphson method [18]. Since these methods are well-studied and are suitable for quasi-convex optimization problems, we will not expand our discussion on this subject in this paper.

\section{B. Maximum Sum Capacity}

In this subsection, we optimize the PS factor by maximizing the sum capacity in both directions. Hence, the problem is formulated as

$$
\rho_{j}^{*}=\underset{\rho_{j} \in[0,1]}{\arg \max }\left(C_{S_{1}}^{(j)}+C_{S_{2}}^{(j)}\right) .
$$

Note that the conclusion drawn from Theorem 1 is not applicable to the maximum sum capacity RS. Although we can always apply the same optimization algorithm to this problem, the solution is not necessarily the global optimum. However, through our simulations in Section V, we find that the obtained solutions almost always yield good results.

\section{Relay SELECTiON}

To achieve different goals, we present the following two RS criteria. The outage probability captures the fairness of the selection scheme and the sum capacity criteria captures the overall network performance. Note that for these criteria, the optimization of the PS factor is independent from relay selection. Therefore, the joint optimization is equivalent to first optimize the $\rho_{j}$ for each relay and then select the best relay.

With the optimal PS factor $\rho_{j}^{*}$ at each relay candidate that maximizes the minimum SINR if $R_{j}$ is selected, we can then select the relay that achieves the maximum worse SINR among all the candidates. The RS problem with minimum outage probability can be formulated as

$$
j_{O P}^{*}=\underset{j \in\{1,2, \cdots, N\}}{\arg \max } \max _{\rho_{j} \in[0,1]} \min \left(\gamma_{S_{1}}^{(j)}, \gamma_{S_{2}}^{(j)}\right) .
$$

Similarly, the RS scheme to achieve the maximum sum capacity can be formulated as

$$
j_{S C}^{*}=\underset{j \in\{1,2, \cdots, N\}}{\arg \max } \max _{\rho_{j} \in(0,1)}\left(C_{S_{1}}^{(j)}+C_{S_{2}}^{(j)}\right) .
$$

\section{Simulations}

In this section, we present the simulations to evaluate the performance and characteristics of the proposed RS schemes. Simulation parameters are set as in Table I and all channels follow Rayleigh fading. As for the residual self-interference channel gain, $-85 \mathrm{~dB}$ to $-15 \mathrm{~dB}$ is considered as a pragmatic range. The self-interference channel gain without cancellation is approximately $-15 \mathrm{~dB}$ [11] and practical self-interference cancellation can already suppress the interference by $70 \mathrm{~dB}$ or more [7]. Therefore, we set $\mathbb{E}\left[\left|\Omega_{S_{i}}\right|^{2}\right]$ to $-85 \mathrm{~dB}$ as the self-interference is unambiguously harmful at the sources. $\mathbb{E}\left[\left|\Omega_{R_{j}}\right|^{2}\right]$ is set to $-85 \mathrm{~dB}$ except for the simulation in Section V-C, where the effect of self-interference cancellation is under investigation.

TABLE I: Simulation Parameters

\begin{tabular}{|c|c|}
\hline Bandwidth & $10 \mathrm{MHz}$ \\
\hline Noise Power Spectral Density & $-174 \mathrm{dBm} / \mathrm{Hz}$ \\
\hline Noise Figure & $5 \mathrm{~dB}$ \\
\hline Energy Harvesting Efficiency & $90 \%$ \\
\hline Residual Self-Interference Channel Gain & $-85 \mathrm{~dB}$ \\
\hline Outage Threshold & $0.5 \mathrm{bps} / \mathrm{Hz}$ \\
\hline
\end{tabular}




$$
\begin{aligned}
\gamma_{S_{i}}^{(j)} & =\frac{\beta_{j}^{2} \rho_{j}\left|h_{i j}\right|^{2}\left|h_{\overline{i j}}\right|^{2} P_{S}}{\left|h_{i j}\right|^{2}\left|\Omega_{R_{j}}\right|^{2} \beta_{j}^{2} \rho_{j} P_{R_{j}}+\left|h_{i j}\right|^{2} \beta_{j}^{2} \rho_{j} \sigma_{A}^{2}+\left|\Omega_{S_{i}}\right|^{2} P_{S}+\left|h_{i j}\right|^{2} \beta_{j}^{2} \sigma_{P}^{2}+\sigma_{S}^{2}} \\
& =\frac{\left|h_{i j}\right|^{2}\left|h_{\bar{i} j}\right|^{2} P_{S}}{\frac{\left(1-\rho_{j}\right) \xi\left|\Omega_{R_{j}}\right|^{2}}{1-\left(1-\rho_{j}\right) \xi\left|\Omega_{R_{j}}\right|^{2}}\left|h_{i j}\right|^{2}\left[P_{S}\left(\left|h_{i j}\right|^{2}+\left|h_{\overline{i j}}\right|^{2}\right)+\sigma_{A}^{2}\right]+\left(\sigma_{A}^{2}+\frac{\sigma_{P}^{2}}{\rho_{j}}\right)\left|h_{i j}\right|^{2}+\frac{\left|\Omega_{S_{i}}\right|^{2} P_{S}+\sigma_{S}^{2}}{\left(1-\rho_{j}\right) \xi}}
\end{aligned}
$$

\section{A. Performance Comparison}

We first investigate the performance of the proposed RS schemes with various source transmit SNR as presented in Figs. 2-4. To demonstrate the effectiveness of our proposed RS schemes, we compare them with the random RS scheme and the all-participate (AP) scheme. For the AP scheme, all the available relays transmit over orthogonal channels with equally allocated bandwidth to avoid the potential inter-relay interference caused by the FD relaying mode. Our proposed RS scheme uniformly outperforms the random RS scheme in terms of both the ergodic sum capacity and the outage probability. In Fig. 3, the outage probability curves of both the proposed RS scheme and the AP scheme have steeper slope than the random RS scheme, meaning they could exploit the cooperative diversity to obtain performance gain.

When the source transmit power is low, the AP scheme performs better than the RS scheme in terms of sum capacity. This is mainly because that the AP scheme could harvest more energy and enhance the overall SINR at the sources. Whereas when the source transmit power is high, selecting the best relay that could utilize the channel bandwidth more efficiently becomes more important, since the best relay can harvest considerable amount of energy by itself already. As a result, the RS scheme outperforms the AP scheme.

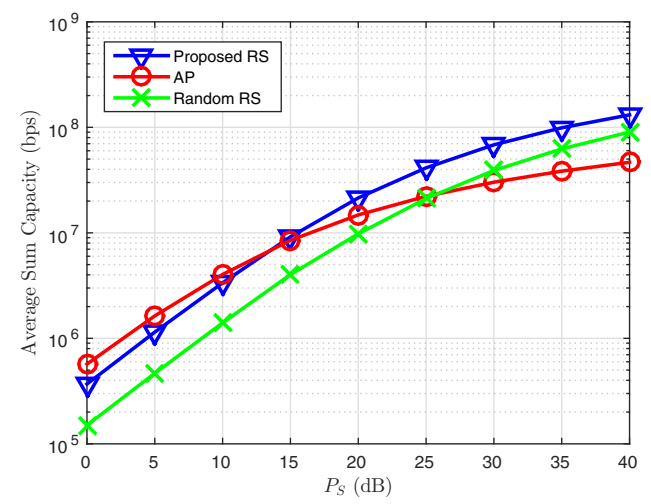

Fig. 2: Sum capacity comparisons with varying source transmission SNR

In Fig. 3, however, the AP scheme yields better outage probability than the proposed RS scheme at high SNR. This is on the contrary to the performance shown in Fig. 2. To further investigate this phenomenon, we plot the empirical cumulative distribution function (CDF) of the minimum capacity of the two directions in Fig. 4. Note that, by definition, the value of the $\mathrm{CDF}$ at $C_{t h}$ is the outage probability if $C_{t h}$ is chosen

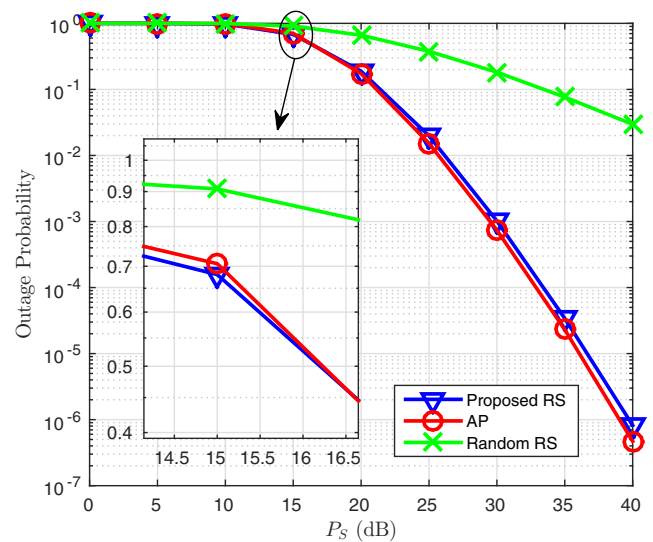

Fig. 3: Outage probability comparisons with varying source transmission SNR

as the threshold. We can see clearly from this figure that the choice of the outage threshold affects the relative relationship of the outage probability of the proposed RS schemes. At the transmit SNR of $25 \mathrm{~dB}$, the proposed RS scheme has higher outage probability than the AP scheme if the threshold is small. The order is reversed if a higher threshold is chosen at the same SNR. The threshold for outage is set to $0.5 \mathrm{bps} / \mathrm{Hz}$ while plotting Fig. 3. And that explains why, at high SNR, the proposed RS scheme yields higher outage probability in Fig. 3 while achieving higher average sum capacity in Fig. 2.

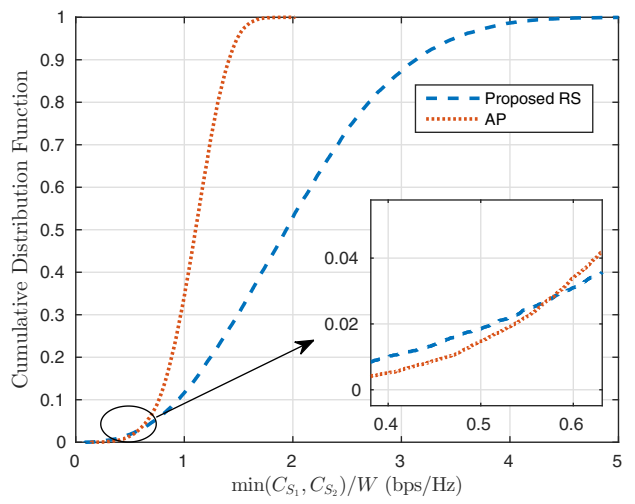

Fig. 4: The cumulative distribution functions of the min capacity at $25 \mathrm{~dB}$.

\section{B. Number of Relays}

The number of available relay candidates that are available for selection also affects the performance of the proposed 
RS schemes. As shown in Fig. 5 and Fig. 6, because of the diversity gain brought by the relays, increasing the number of relay candidates benefits all the proposed RS schemes except the AP one. When the number of available relays is large, selecting all of them causes the bandwidth to be split into too many subbands. As a result, the SINR gain brought by the extra power harvested by the relays is no longer large enough to overcome the capacity loss due to the bandwidth reduction, especially at high SNR.

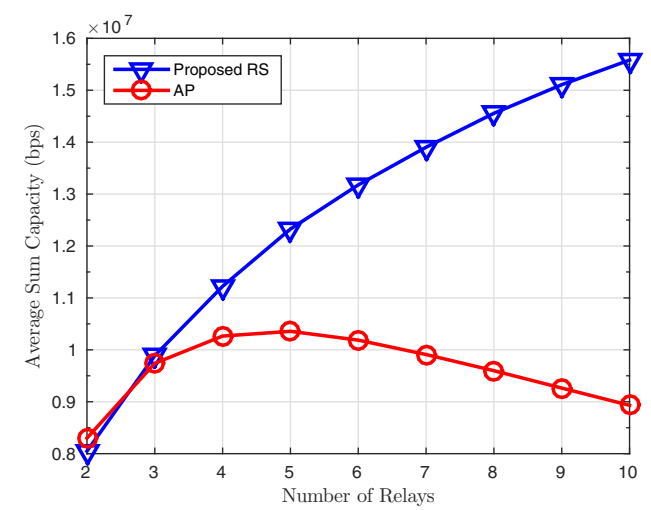

Fig. 5: Sum capacity comparisons with different number of relays

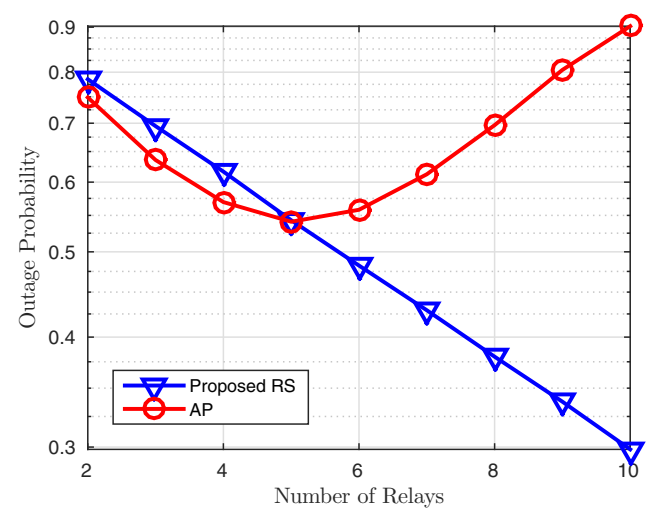

Fig. 6: Outage probability comparisons with different number of relays

\section{Effects of Self-Interference at the Relays}

The sum capacity and the outage probability with different self-interference channel gains are shown in Fig. 7 and Fig. 8, respectively. $\mathbb{E}\left[\left|\Omega_{R_{j}}\right|^{2}\right]$ varies from $-55 \mathrm{~dB}$ to $-15 \mathrm{~dB}$. The transmit SNR is set to $20 \mathrm{~dB}$, with other settings unchanged.

In Fig. 7, we observe that the sum capacity is monotonically increasing when the residual self-interference channel gain becomes weaker. And it begins to plateau when $\mathbb{E}\left[\left|\Omega_{R_{j}}\right|^{2}\right]$ is below $-30 \mathrm{~dB}$. Intuitively, however, there should be a nonzero optimal $\mathbb{E}\left[\left|\Omega_{R_{j}}\right|^{2}\right]$ for such EH relay networks, since the system could benefit from the loopback interference at the relays as an additional source of energy that can be harvested. Nonetheless, as we can see in (16), the end-to-end SINR is

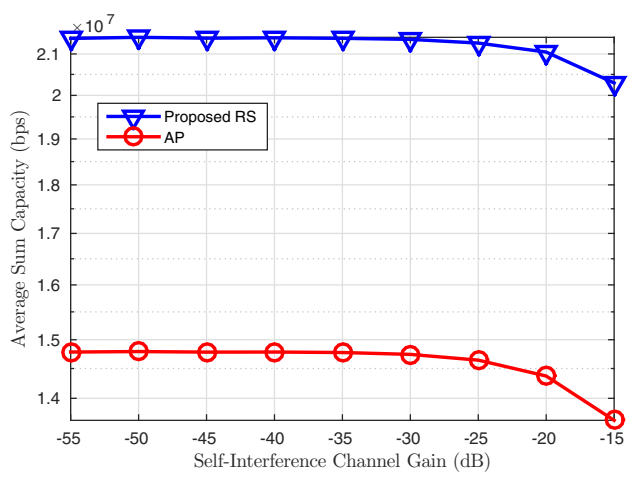

Fig. 7: Sum capacity comparisons with varying residual selfinterference channel gain

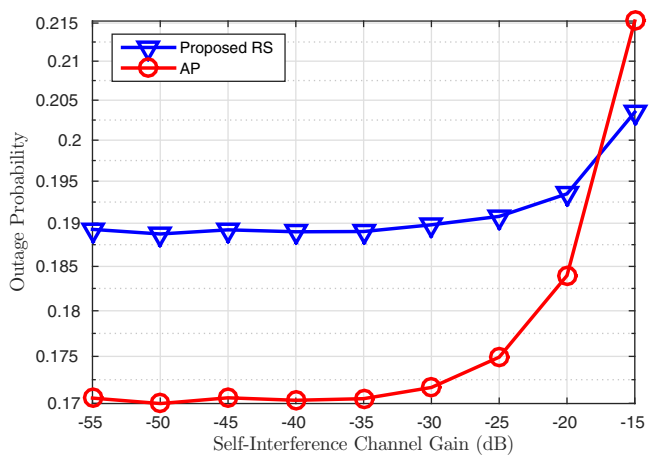

Fig. 8: Outage probability comparisons with varying residual self-interference channel gain

monotonically decreasing with $\mathbb{E}\left[\left|\Omega_{R_{j}}\right|^{2}\right]$. This is caused by the approximation made in (10), which assumes the power of the received signals at the relays is much larger than that of the processing noise. In this case, the power harvested from the self-interference is simply too little to overcome its undesirable effect, namely the SINR degradation at the relays.

In Fig. 8, the outage probability curves of the proposed RS and the AP schemes cross over each other between $-20 \mathrm{~dB}$ and $-15 \mathrm{~dB}$. This phenomenon is absent in the sum capacity performance in Fig. 7. The reason is similar to that explained in Section V-A. That is the behavior of the outage probability is highly sensitive to the threshold of outage capacity, especially for the AP scheme whose capacity distribution is more concentrated around its mean than the proposed RS scheme.

\section{CONCLUSIONS}

In this paper, we introduced PS-based SWIPT into twoway full-duplex relaying networks. The energy carried by the signals transmitted from the sources are partially harvested by the cooperating relays. The harvested energy at the relays is then used to amplify and forward the combined signals back to the sources. The quasi-convexity of the PS factor optimization was proved, and the optimal PS factor for each relay was obtained by one-dimensional search. In order to achieve the minimum outage probability and the maximum sum capacity, 
respectively, we proposed two efficient RS schemes based on the optimized PS factor at each relay. Both of the proposed RS schemes outperform the random RS scheme. At high SNR, the proposed RS schemes also achieve higher sum capacity than the AP scheme.

\section{ACKNOWLEDGMENT}

This work was supported in part by the National Science Foundation under grant number CNS-1343189.

\section{APPENDIX}

PROOF OF THEOREM 1

Proof: Let $\tilde{\gamma}_{S_{i}}^{(j)}$ denote the denominator in (16), so that $\gamma_{S_{i}}^{(j)}=\left|h_{i j}\right|^{2}\left|h_{\bar{i} j}\right|^{2} P_{S} / \tilde{\gamma}_{S_{i}}^{(j)}$.

$$
\begin{aligned}
J_{P_{\text {out }}}^{(j)} & =\max \left(-\gamma_{S_{1}}^{(j)},-\gamma_{S_{2}}^{(j)}\right) \\
& =\max \left(-\frac{\left|h_{1 j}\right|^{2}\left|h_{2 j}\right|^{2} P_{S}}{\tilde{\gamma}_{S_{1}}^{(j)}},-\frac{\left|h_{2 j}\right|^{2}\left|h_{1 j}\right|^{2} P_{S}}{\tilde{\gamma}_{S_{2}}^{(j)}}\right)
\end{aligned}
$$

Since both maximization and composition with a nondecreasing function preserve quasi-convexity, and $f(x)=$ $-\frac{\left|h_{i j}\right|^{2}\left|h_{\bar{i} j}\right|^{2} P_{i}}{x}$ is a non-decreasing function for $x>0, J_{P_{\text {out }}}^{(j)}$ is quasi-convex if both $\tilde{\gamma}_{S_{1}}^{(j)}$ and $\tilde{\gamma}_{S_{2}}^{(j)}$ are positive and quasiconvex.

Since $\left(1-\rho_{j}\right) \xi\left|\Omega_{R_{j}}\right|^{2} \ll 1$, we can approximate $\tilde{\gamma}_{S_{i}}^{(j)}$ as

$$
\begin{aligned}
\tilde{\gamma}_{S_{i}}^{(j)} \approx & \left(1-\rho_{j}\right) \xi\left|\Omega_{R_{j}}\right|^{2}\left|h_{i j}\right|^{2}\left(P_{S}\left(\left|h_{i j}\right|^{2}+\left|h_{\bar{i} j}\right|^{2}\right)+\sigma_{A}^{2}\right) \\
& +\left(\sigma_{A}^{2}+\frac{\sigma_{P}^{2}}{\rho_{j}}\right)\left|h_{i j}\right|^{2}+\frac{\left|\Omega_{S_{i}}\right|^{2} P_{S}+\sigma_{S}^{2}}{(1-\rho) \xi} .
\end{aligned}
$$

$\tilde{\gamma}_{S_{i}}^{(j)}$ is obviously positive because it is the denominator of the SINR. To prove that it is quasi-convex, we derive its second derivative as

$$
\frac{\partial^{2} \tilde{\gamma}_{S_{i}}^{(j)}}{\partial \rho_{j}^{2}} \approx \frac{2 \sigma_{P}^{2}\left|h_{i j}\right|^{2}}{\rho_{j}^{3}}+\frac{2\left(\left|\Omega_{S_{i}}\right|^{2} P_{S}+\sigma_{S}^{2}\right)}{\left(1-\rho_{j}\right)^{3} \xi} .
$$

It is obvious that $\frac{\partial^{2} \tilde{\gamma}_{S_{i}}^{(j)}}{\partial \rho_{j}^{2}}>0$ for $\rho_{j} \in(0,1)$. Consequently, $\tilde{\gamma}_{S_{i}}^{(j)}$ is convex. And a convex function is also quasi-convex. Therefore, $\tilde{\gamma}_{S_{i}}^{(j)}$ is quasi-convex and that concludes the proof.

\section{REFERENCES}

[1] L. Varshney, "Transporting information and energy simultaneously," in Proc. Intl. Symp. on Information Theory, 2008, pp. 1612-1616.

[2] P. Grover and A. Sahai, "Shannon meets Tesla: Wireless information and power transfer," in Proc. Intl. Symp. on Information Theory, 2010, pp. 2363-2367.

[3] X. Zhou, R. Zhang, and C. K. Ho, "Wireless information and power transfer: Architecture design and rate-energy tradeoff," IEEE Trans. on Communications, vol. 61, no. 11, pp. 4754-4767, November 2013.

[4] A. Nasir, X. Zhou, S. Durrani, and R. Kennedy, "Relaying protocols for wireless energy harvesting and information processing," IEEE Trans. on Wireless Communications, vol. 12, no. 7, pp. 3622-3636, July 2013.
[5] M. Duarte and A. Sabharwal, "Full-duplex wireless communications using off-the-shelf radios: Feasibility and first results," in Proc. Asilomar Conf. on Signals, Systems, and Computers, 2010, pp. 1558-1562.

[6] M. Duarte, C. Dick, and A. Sabharwal, "Experimentdriven characterization of full-duplex wireless systems," IEEE Trans. on Wireless Communications, vol. 11, no. 12, pp. 4296-4307, December 2012.

[7] S. Li and R. Murch, "An investigation into baseband techniques for single-channel full-duplex wireless communication systems," IEEE Trans. on Wireless Communications, vol. 13, no. 9, pp. 4794-4806, September 2014.

[8] N. Shende, O. Gurbuz, and E. Erkip, "Half-duplex or full-duplex relaying: A capacity analysis under selfinterference," in Annual Conference on Information Sciences and Systems (CISS), 2013, pp. 1-6.

[9] C. Zhong, H. Suraweera, G. Zheng, I. Krikidis, and Z. Zhang, "Wireless information and power transfer with full duplex relaying," IEEE Trans. on Communications, vol. 62, no. 10, pp. 3447-3461, October 2014.

[10] M. Mohammadi, H. Suraweera, G. Zheng, C. Zhong, and I. Krikidis, "Full-duplex MIMO relaying powered by wireless energy transfer," in IEEE International Workshop on Signal Processing Advances in Wireless Communications (SPAWC), 2015, pp. 296-300.

[11] Y. Zeng and R. Zhang, "Full-duplex wireless-powered relay with self-energy recycling," IEEE Wireless Communications Letters, vol. 4, no. 2, pp. 201-204, April 2015.

[12] D. Wang, R. Zhang, X. Cheng, and L. Yang, "Capacityenhancing full-duplex relay networks based on power splitting (PS-)SWIPT," IEEE Trans. on Vehicular Tech., 2016 (submitted).

[13] H. Cui, M. Ma, L. Song, and B. Jiao, "Relay selection for two-way full duplex relay networks with amplify-andforward protocol," IEEE Trans. on Wireless Communications, vol. 13, no. 7, pp. 3768-3777, July 2014.

[14] L. Song, "Relay selection for two-way relaying with amplify-and-forward protocols," IEEE Trans. on Vehicular Tech., vol. 60, no. 4, pp. 1954-1959, May 2011.

[15] S. Atapattu, Y. Jing, H. Jiang, and C. Tellambura, "Relay selection schemes and performance analysis approximations for two-way networks," IEEE Trans. on Communications, vol. 61, no. 3, pp. 987-998, March 2013.

[16] K.-H. Liu, "Outage-optimal relay selection for energyharvesting relays based on power splitting," in International Conf. on Wireless Communications \& Signal Processing (WCSP), Nanjing, China, October 15-17, 2015, pp. 1-6.

[17] J. Men, J. Ge, C. Zhang, and J. Li, "Joint optimal power allocation and relay selection scheme in energy harvesting asymmetric two-way relaying system," IET Communications, vol. 9, no. 11, pp. 1421-1426, July 2015.

[18] E. Chong and S. Żak, An Introduction to Optimization, 2nd ed. Wiley, 2001. 